\title{
Allergic Sensitization and Rhinitis in Children: What Is New?
}

\section{Désirée Larenas-Linnemann, MD}

\author{
Address \\ Hospital Médica Sur, Torre 2, cons.602, Puente de Piedra 150, Colonia Toriello \\ Guerra, Delegación Tlalpan, 14050, Mexico, DF, Mexico \\ Email: marlar1@prodigy.net.mx
}

Published online: 28 January 2015

(C) Springer International Publishing AG 2015

This article is part of the Topical Collection on Allergic Rhinitis

Keywords Allergic rhinitis - Pediatric - House dust mite · Pollens · Skin prick test - Immunotherapy

\section{Opinion statement}

In children with nasal symptoms during several months, oftentimes with a chronically relapsing course, allergic rhinitis is one of the most probable diagnoses. This is often complicated with recurrent respiratory infections, as there is a circular causal loop between allergic rhinitis that increases the frequency of respiratory infections and the infections that exacerbate the allergic disease. As a consequence, in these children, the physician should intentionally search for allergic sensitization, in serologic specific IgE testing and/or with skin prick testing. If a possible allergic diathesis is further suspected because of a personal (atopic dermatitis) or a family history of allergic diseases, the search for allergic sensitization should begin at an even earlier age; in several cases, allergic sensitization can already be shown even before the age of two. In my opinion, in highly atopic children as soon as a specific allergic sensitization can be documented that coincide with the presence of symptoms on possible exposure, as obtained from the detailed history, allergen immunotherapy should be offered as an integral part of the treatment. It is very well plausible that the earlier in the development of the allergic disease, we can re-direct the immune system, the better the results. However, in these young allergic children, I only choose sublingual immunotherapy (SLIT), being the option with the best safety profile. In our clinic, we have looked for several ways to improve adherence, which is an issue especially in SLIT. Among the several strategies we implemented are written information on the treatment and the administration schedule with a 20 min explanation by a specialized nurse at start, a flexible, 1-month up-dosing phase to reduce the frequency and intensity of local side effects and reduce the frequently seen flare in nasal symptoms, morning dosing, up-dosing under the cover of an antihistamine and a topical corticosteroid in the evening for 6 weeks, a close follow-up of the children by phone contacts, and an initial follow-up visit at 6 weeks. 


\section{Introduction}

The vast majority of chronic rhinitis patients among the pediatric age group suffer from allergic rhinitis. As described in the international literature and just recently confirmed for my own country-México-as well, the primary sensitization in young children is to the house dust mite, Dermatophagoides [1]. Moreover, in these children, allergic rhinitis is often of the perennial, intermittent type. Also, it is accompanied in more than half of the children by allergic, mild-moderate asthma [2].

The treatment of rhinitis in the child with allergic sensitization starts with the removal of the allergen(s) the child is allergic to, to reduce ongoing inflammation as best as possible [3, 4]. Environmental control measures are most useful when installing several at a time, e.g., for a house dust mite-sensitized child, these could include removal of carpet, furnished upholstering, improve ventilation, keeping furry toys in a closed cage, and mattress encasement [3,5]. However, most allergens are difficult to avoid completely; thus, environmental control measures can only partially reduce symptoms. In a second step, symptoms should be controlled with medication: antihistamines (systemic or local) are useful when rhinorrhea, sneezing, and pruritus are the main complaints, but when the disorder is more severe, long-standing, or the symptoms include nasal obstruction, it is generally necessary to add topical corticosteroids to the treatment [6]. In children, rarely short courses of systemic steroids are needed and depot corticosteroids should be avoided at all costs.
As for oral antihistamines the right group of medication should be selected: first-generation, sedating antihistamines can have deleterious effects on school performance and quality of sleep. Most new secondgeneration antihistamines are almost devoid of CNS penetration and thus should be the group of oral $\mathrm{H} 1$ antihistamines prescribed. Among the secondgeneration antihistamines, there is still a difference in CNS penetration and-especially for children-those with hardly any sedative effect would be best, although some are so new that till now only approval for the 12-year and higher age groups has been obtained.

As for corticosteroids, those with the lowest bioavailability should be selected in children.

Finally, many children with an atopic background shall eventually continue the allergic march. Till now, allergen immunotherapy (AIT) is the only treatment directed at the underlying cause of allergy. As the sublingual route offers a non-invasive method with a good safety profile, the World Allergy Organization (WAO) guidelines on sublingual immunotherapy recommend starting SLIT in the early stages of the disease. To obtain the best effect, the sooner the treatment is started-once a clear sensitization is documented-the better. And to obtain a longlasting effect from AIT, it is recommended for a duration of at least 3 years, but this recommendation is based on adult trials [7].

\section{Treatment options for pediatric allergic rhinitis}

Although there are other treatment options for allergic rhinitis in children, see Table 1, the focus of this paper shall be on allergic sensitization and the treatment related to that: allergen immunotherapy.

\section{Allergic sensitization and avoidance}

Allergen avoidance is the first step in the management of any allergic disorder. For pediatric allergic rhinitis, sensitization profiles have been reported for some study populations. Italian investigators [9] did skin prick test (SPT) in over 1300 children and found that $6.2 \%$ of the patients were pollen-monosensitized, and $84.9 \%$ were sensitized to $\geq 3$ pollens. Moreover, they showed that a longer allergic rhinitis (AR) duration was significantly associated with moderate-tosevere AR symptoms ( $p=0.004)$, asthma $(p=0.030)$, and oral allergy syndrome $(p<0.001)$. 


\section{Table 1. Treatment options for allergic rhinitis in children}

\begin{tabular}{|c|c|c|}
\hline Management options & Specific interventions & Comments \\
\hline \multirow[t]{2}{*}{ Allergen avoidance } & $\begin{array}{l}\text { HDM: mattress encasements eliminate } \\
\text { upholstered furniture, carpets, furry toys }\end{array}$ & $\begin{array}{l}\text { For house dust mite, only multiple intervention } \\
\text { trials might show some benefit [3] }\end{array}$ \\
\hline & $\begin{array}{l}\text { Cat/dog: take the animal out of the house } \\
\text { Rodents: pest control and other } \\
\text { environmental control measures. }\end{array}$ & $\begin{array}{l}\text { Animal dander and rodent allergy: avoidance of } \\
\text { the animal(s), as complete as possible }[4,8]\end{array}$ \\
\hline \multirow[t]{3}{*}{ Topic medication } & $\begin{array}{l}\text { Nasal washes: saline, isotonic, hypotonic, } \\
\text { hypertonic } \\
\text { Intranasal antihistamines }\end{array}$ & \\
\hline & Intranasal corticosteroids & \\
\hline & $\begin{array}{l}\text { Intranasal combination: antiH+CS } \\
\text { (not yet studied in children) }\end{array}$ & \\
\hline \multirow[t]{2}{*}{ Systemic medication } & $\begin{array}{l}\text { Intranasal immunotherapy: No } \\
\text { Antihistamines (second-generation, non- } \\
\text { sedating) } \\
\text { Antileucotrienos }\end{array}$ & $\begin{array}{l}\text { Antileukotriene receptor antagonists only in } \\
\text { combination treatment with topical CS or } \\
\text { antihistamines. }\end{array}$ \\
\hline & $\begin{array}{l}\text { Combinations: antihistamine- } \\
\text { antileukotriene } \\
\text { - Systemic corticosteroids }\end{array}$ & $\begin{array}{l}\text { Systemic corticosteroids only indicated in severe } \\
\text { exacerbations for a short course (5-7 days). } \\
\text { Depot systemic corticosteroids are contra- } \\
\text { indicated }\end{array}$ \\
\hline $\begin{array}{l}\text { Allergen } \\
\text { immunotherapy }\end{array}$ & $\begin{array}{l}\text { Subcutaneous AIT } \\
\text { Sublingual AIT }\end{array}$ & \\
\hline
\end{tabular}

In Mexico, the main sensitizing allergen in allergic rhinitis in children and adults was the house dust mite Dermatophagoides pteronyssinus in all climatological zones, from the dry North of the country till the humid southeastern areas where house dust mite (HDM) sensitization reached almost $90 \%$. Grass pollen sensitization was to the tropical grass, Cynodon dactylon, and ash and oak pollen were the main tree allergens. The only exception is the central part of the country, where agriculture is one of the main income sources and grass pollen is the prime sensitizer [1]. In this epidemiological study, children had mostly intermittent moderate-severe, perennial allergic rhinitis and asthma was a comorbid condition in more than half of the children [2].

A retrospective review of all children diagnosed with chronic rhinosinusitis in a large tertiary-care pediatric hospital in Boston over a 10-year period showed that $26.9 \%$ had allergic rhinitis. As a consequence, the authors strongly recommend to consider formal allergy testing, guided by clinical history and regional allergen sensitivity prevalence, in all children with chronic rhinosinusitis (CRS) and in particular in those with reactive airway disease [10]. These investigators went further on to determine the sensitization profiles of the children with CRS and AR and found this was very similar to that reported previously for children with only AR: indoor aeroallergen sensitivities (63$100 \%$ ) were more prevalent than outdoor aeroallergen sensitivities (44-50\%) and the most common indoor aeroallergen sensitivity was to dust mites (50- 
$75 \%)$ and the outdoor sensitivity was mostly to tree pollen [11].

Although allergen avoidance is the first recommended action step, it has been hard till now to show a clear positive impact of HDM exposure constraint measures. That is why the Practice Parameters on Environmental Control for house dust mite allergy makes a suggestion to implement HDM avoidance measures, without strongly recommending it. Also, the Parameters stresses that the best results shall probably be obtained when a combination of various methods is applied [3].

In another perspective, interesting results of an epidemiologic survey in 292 children with acute lymphoblastic leukemia and the presence or absence of allergy and allergic sensitization was reported by a group of Greek investigators. They showed that a self-reported-allergic history overall (odds ratio (OR) 0.49, $95 \%$ confidence interval (CI) $0.34-0.72$ ) and practically each one of its main components (respiratory, food, any other clinical allergy) were strongly and inversely associated with ALL. Likewise, the serum IgE inverse association was of the same magnitude (OR 0.43, $95 \%$ CI 0.22-0.84) mainly contributed by food IgE (OR 0.39, $95 \%$ CI 0.18-0.83) [12].

\section{Correct diagnosis}

For correct and effective AIT, both SLIT and subcutaneous immunotherapy (SCIT), the correct patient selection is crucial. For the selection of the optimal candidates for AIT in the first place, a detailed clinical history should be taken, in which the physician-expert in allergic diseases-intentionally looks for activation of symptoms in relation to a probable exposure to the allergen(s).

Generally, the design of clinical trials takes good care of the correct patient selection in the inclusion and exclusion criteria and patients are only selected to be recruited when they have at least a certain symptom score during the run-in phase. In some trials, even an observational year precedes the treatment year. However, this is not always the case, as was shown by recently published study outcomes in the Journal of Negative Results: a phase III trial of sublingual immunotherapy with a 2800 BAU grass tablet in adult patients with grass pollen-induced allergic rhinitis, with or without conjunctivitis, with or without asthma, did not demonstrate significant symptom improvement versus placebo. Lack of a relationship between pollen count and symptom scores in the population included and post hoc findings of symptom scores among subjects with low pre-seasonal symptoms suggest that the symptoms reported in this study were not primarily reflective of the effects of grass pollen exposure [13].

After this first phase, purely based on history, which ends with the suspicion of causal allergen(s) per patient, the presence of specific IgE has to be demonstrated. Nowadays, there are several methods for showing the presence of specific IgE, but the skin prick test with high-quality extracts is still mostly used. Recent findings showed in adults a better treatment effect in the group with both SPT and serum IgE positivity [7] and preliminary data of a pilot study in children with house dust mite allergy documented results in this same direction: the best results of a 3-year course of SLIT were seen in the group that had serum sIgE levels for HDM $>10 \mathrm{kU} / \mathrm{l}$ as compared to those with a serum sIgE below that limit [14]. 


\section{Primary and secondary trial outcome measures}

One last issue to consider, before discussing trial results, is related to the primary and secondary outcome measures selected in the trials. As was recently debated $[15,16]$, it is not easy to capture the complete impact of AIT in simple measures, and it is even harder to do so in pediatric trials, where the subjective outcome measures generally shall be reported by a third party: the caretaker. The complete effect of AIT goes beyond a simple lowering of allergic symptoms and medication score, as allergy affects the general well-being. As such, lately, several new outcome measures have been tested, more related to the general well-being, e.g., the quality of life questionnaires. Dutch investigators analyzed the pediatric and adolescent disease-specific rhinoconjunctivitis quality of life questionnaire, concluding they are relevant, but the investigators suggest they could be shortened as there is a substantial number of irrelevant items in the original questionnaires. They also analyzed the usefulness of the generic COOP/WONCA charts finding them not relevant in the monitoring of children and adolescents with allergic rhinoconjunctivitis caused by grass pollen. Finally, the investigators' opinion concerning the retrospective global assessment of symptoms (GAS) is that it does not sufficiently reflect the actual symptoms during the preceding season [17].

That allergen-specific bronchial provocation testing might be a useful method to assess the efficacy of AIT was reported in house dust mite allergic asthmatic children. After SCIT, the patients' allergen-specific bronchial hyperreactivity (BHR) was significantly improved (PD20 FEV1 before SCIT: 34.4 and after 63.3 AU $(p<0.01))$, while this parameter remained unchanged in the control group. Moreover, the team was able to differentiate between the responders ( $n=17,60.7 \%)$ and non-responders ( $n=11$, no improvement in BAP). The patients in both groups stated that SCIT had led to a subjective improvement in their symptoms, in contrast to the untreated control group, but only the responders required less medication after SCIT $(p<0.01)$. As the study was conducted in a small group of patients, the results need confirmation in a larger trial [18].

Other objective outcomes measures are the biomarkers. After 6 months of HDM SLIT in 116 children with allergic rhinitis, only the well-controlled group showed a statistically significant decrease in IL-5 and TIM-1 and an increase in IL-10 compared to baseline [19]. An in-depth discussion of several other promising biomarkers [20-23] goes beyond the scope of this review.

Apart from the use of correct outcome measures, the inclusion of a randomly assigned control group is crucial, as was shown by Narkus et al. when investigating the placebo effect in AIT trials. The mean placebo effect in grouped adult SCIT trials was up to $41 \%$ in the second treatment year, but only $1 \%$ in the SLIT trial [24]. However, no such data have been published till now in the pediatric age group.

\section{Efficacy in SCIT trials}

A complete review of the latest literature consisting of randomized controlled and open controlled trials on SCIT in the pediatric population was published in 2011 [25]. All publications on SCIT in pediatric patients from January 2006 onward were reviewed. Study design was not a restriction for articles to be included, but their scientific quality was evaluated using the Grading of 
Recommendations Assessment, Development, and Evaluation and Jadad tools. Clinical, safety, and immunologic data were gathered. The scientific evidence produced by the 31 articles analyzed showed that there is high-quality evidence that grass pollen SCIT causes a reduction in the combined symptommedication score and increases the threshold of the conjunctival provocation test after termination of SCIT and still 7 years later. At the same time, the threshold of the specific bronchial provocation test and the skin prick test reactivity were improved. As for Alternaria SCIT, the reviewed trials in children showed improvement in medication scores, combined symptom-medication scores, and quality of life. Alternaria SCIT further augments the threshold in the nasal provocation test. High-quality evidence of house dust mite SCIT shows that asthma symptom and medication scores improve and emergency department visits and skin reactivity are reduced; moderate evidence indicates improvement in pulmonary function tests. Pollen SCIT prevents asthma (moderate evidence); evidence for long-term benefit of pollen SCIT (7-12 years after termination) is low to moderate. However, in the articles reviewed, there was inconclusive evidence for SCIT reducing new sensitizations. These data led the investigators to conclude that there is acceptable evidence that shows that grass pollen, Alternaria, and house dust mite SCIT are beneficial in allergic children.

In 2013, another group of investigators conducted a systematic review on SCIT and SLIT in children. In the 13 randomized controlled trials they found, with in total 920 children in the active plus control groups, the strength of evidence is moderate that SCIT improves asthma and rhinitis symptoms and low that SCIT improves conjunctivitis symptoms and asthma medication scores [26].

Outside of these systematic reviews, very few new randomized trials on pediatric SCIT have been published. Over the past years, the inner-city asthma consortium has been studying the causes of asthma morbidity in children living in urban environments, one of the key contributors being cockroach allergy. As such, they sought to document immune responses to cockroach allergen and to provide direction for the development of immunotherapy for cockroach allergy, conducting four pilot studies. The aim of the consortium is to finally propose treatment options directed at the allergic cause of inner-city asthma in children. First in an open-label study, the safety of cockroach SLIT in adults and children was assessed; then, they sought for biomarkers of cockroach SLIT versus placebo in a randomized, double-blind study in adults; they found a significantly greater increase in cockroach-specific IgE levels between the active and placebo groups (geometric mean ratio, 1.92; $P<0.0001$ ) and a trend toward increased cockroach-specific IgG4 levels in actively treated subjects $(P=0.09)$, but they were not able to detect any evidence of a functional blocking antibody response. Thirdly, a randomized, double-blind biomarker study of two doses of cockroach SLIT versus placebo in children was conducted (5-17 years, $N=99$;), reporting significant differences in IgE, IgG, and IgG4 responses between both active groups and the placebo group, although no consistent differences between the high- and low-dose groups could be shown.

As the results obtained with SLIT were not optimal, the investigators now proceeded with an open-label safety and biomarker study of cockroach SCIT in adults, with the intention to then move on to this treatment in children.

Contrary to the SLIT trial, in this last SCIT trial the treatment resulted in significant changes from baseline in cockroach $\operatorname{IgE}, \operatorname{IgG}_{4}$, and blocking antibody 
levels. SCIT is immunologically more active than SLIT. In all studies, the safety profile of cockroach immunotherapy was reassuring.

\section{Safety of pediatric SCIT}

No major issues concerning SCIT safety in children have been published lately. Preliminary reports of a survey conducted among members of the American Academy of Allergy, Asthma and Immunology showed that SCIT is given before the age of 5 by a third of the respondents, with a total experience of the respondents in this age group of over 2000 children and a reported rate of major problems that led to the interruption of SCIT for medical reasons below $1 \%$ [27].

In Great Britain National Health Service (NHS), AIT practice patterns of pediatric clinics were audited against national immunotherapy guidelines. Contrary to the British AIT guideline recommendations, $55 \%$ of the treated children had asthma. Three quarters of these asthmatic children had asthma treatment at step 2 BTS/SIGN or above. Although adverse events were seen in almost half of the SCIT cycles, they were generally mild and not related in frequency nor in severity to the presence of asthma. Local adverse reactions in SLIT were reported in almost half of the children. Only $3 \%$ interrupted AIT because of $\mathrm{AE}$ [28].

In an attempt to unify the method of reporting of systemic adverse reactions to AIT, a group of experts published the WAO grading system of systemic adverse reactions to AIT [29]. This is not a specific pediatric system though, and its practical aspects still have to be improved further to make it widely applicable in practice.

Lately, two systematic reviews of SLIT in children were published. The first one included all clinical trials of SLIT in the treatment of respiratory and food allergy in patients 18 years or younger, irrespective of their design. Once again, the quality of the scientific evidence from the trials was evaluated with GRADE. Both, clinical outcomes and immunologic changes were tabulated. Results: 29 of the 56 reviewed articles met the inclusion criteria. According to the investigators, new evidence is robust for the efficacy of the pre-coseasonal tablet and the drop grass pollen SLIT in allergic rhinitis in children and scarce for seasonal asthma. Some evidence for Alternaria SLIT efficacy is appearing. For house dust mite (HDM) SLIT in asthma, there is high-quality evidence for medication reduction while maintaining symptom control; evidence for HDM SLIT efficacy in allergic rhinitis is of moderate-low quality. There is moderate evidence for efficacy of dual grass pollen-HDM SLIT after 12 months of treatment, an effect still present 1 year after discontinuation. Specific provocation test results (nasal, skin) improve with grass pollen and HDM SLIT, but nonspecific bronchial provocation testing does not. No anaphylaxis was found among 2469 treated children.

The conclusions of the second systematic review from 2013, already mentioned above, were similar. Here, only randomized pediatric trials were included, summing up to a total of 18 pediatric SLIT studies (1583 children). According to the investigators, there was high-quality evidence that SLIT improves asthma symptoms and moderate-quality evidence that SLIT improves 
rhinitis and conjunctivitis symptoms and decreases medication usage. In the three included head-to-head comparison of SCIT vs. SLIT, the evidence is low to support SCIT over SLIT for improving asthma or rhinitis symptoms or medication usage [26].

\section{Safety of pediatric SLIT}

Although there had already been several reports of eosinophilic esophagitis as an adverse reaction secondary to oral immunotherapy with certain foods [30], there has now been one report of a confirmed eosinophilic esophagitis secondary to grass pollen SLIT, be it in an adult patient [31]. Just as for SCIT, the need for a uniform method of reporting the adverse reactions to SLIT led to the construction of a WAO grading system for the reporting of local adverse reactions, published by Passalacqua et al. [32]

\section{Allergen immunotherapy efficacy in real life}

To apply AIT in the clinic and translate data obtained in clinical trials to day-to-day practice apart from the above, several other issues have to be taken into account.

1. Quality of the extracts

Although the pooling of data on all trials conducted with SCIT or SLIT improves the quality of evidence of the data by augmenting the total number of patients treated and well-conducted systematic reviews may enhance the scientific quality of conclusions, there is a certain danger inherent to this kind of analysis: the over-generalization of the results. Pooling all data from well-conducted clinical trials and using the outcome of this kind of analysis to conclude on the efficacy of all SCIT or SLIT do not give the needed importance to the quality of products used to conduct the trials. Although a highly concentrated sublingual drop extract can have positive outcomes, this does not allow us to conclude that all sublingual drops, whatever their concentration, can be effective. Thus, the conclusions drawn from SLIT and SCIT trials hold only true for the studied products.

2. Issues of mono- and multisensitization and mono-multi allergy

The efficacy of AIT in clinical trials has almost exclusively been shown for AIT with one allergen only (mono-allergen immunotherapy), both for SCIT and SLIT. However, in many parts of the world, patients are often polysensitized [1] and poly-allergic. One of the very few trials, if not the only, showing multiallergen SCIT is effective in children dates back more than 50 years [33]. For SLIT, the efficacy of dual-allergen immunotherapy in HDM pollen dual allergic children and adults was documented with positive changes in both, clinical and immunological parameters [34]. However, several years ago, a 10-allergen mix had been found of no use, even though the adequate concentration of the study allergen was maintained [35].

In this sense, the expert should bear in mind to carefully select only those very few allergens of importance in the patient's pathology and to only combine a minimal number of allergens in one vial, especially for SLIT.

\section{Compliance}

One of the major problems with AIT in everyday practice is the low compliance rate. This had already been pointed out by Hankin et al. when reviewing Florida Medicaid data [36]. It was also very recently pointed out by Dutch 


\section{Table 2. Immunotherapy adherence issues}

\begin{tabular}{|c|c|}
\hline Impediments with adherence: & Ways to Improve Adherence \\
\hline \multicolumn{2}{|r|}{ 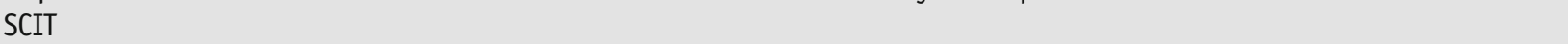 } \\
\hline $\begin{array}{l}\text { 1. Inconvenience having to go to a health care } \\
\text { provider's office for administration }\end{array}$ & $\begin{array}{l}\text { 1. Education and Dialogue with patient, parent, and/or } \\
\text { caregiver prior to start of immunotherapy }\end{array}$ \\
\hline 2. Risk of anaphylaxis & a. What is allergen immunotherapy? \\
\hline 3. Requires needle injection & b. How does AIT work? \\
\hline 4. Time required to see clinical improvement & c. Benefits and risks of AIT \\
\hline 5. Time loss from work or school to receive SCIT & d. Time commitment to treatment \\
\hline 6. Cost issues & e. Which is best for the patient, SCIT vs SLIT \\
\hline \multicolumn{2}{|l|}{ Impediments with adherence: } \\
\hline SLIT & 2. Cost issues \\
\hline 1. Inconvenience of daily treatment & a. Government and Insurance coverage \\
\hline 2. Local side effects especially mouth and GI & b. Out of pocket costs for the patient \\
\hline 3. Time required to see clinical improvement & 3. Follow-up after initiation of immunotherapy \\
\hline 4. Cost issues & a. Frequent office visits \\
\hline & b. Follow-up phone calls by a nurse \\
\hline
\end{tabular}

investigators: overall, only $18 \%$ of AIT children and adolescents reached the minimally required duration of treatment of 3 years. The numbers were somewhat better for SCIT - 23\%-than for SLIT-7 \%. The median durations for SCIT and SLIT users were 1.7 and 0.6 years, respectively $(P<0.001)$. In this study, it was pointed out that a longer persistence on AIT treatment was related to the prescriber (longer if AIT was prescribed by a family physician vs. allergist), whereas a shorter persistence rate was related to single-allergen immunotherapy, lower socioeconomic status, and younger age [37]. Patient adherence is also related to economic stability of the population in general. During the financial crisis in Spain, patients have been less compliant with AIT [38].

Recent findings agree on the fact that some interventions (i.e., patients' education, strict follow-up, regular contacts) could effectively improve the adherence [39] and the following Table 2 with actions to improve AIT adherence was proposed [40].

\section{Pharmacoeconomics of allergen immunotherapy}

A positive finding from the same study by Hankin et al. relates to the total health-care costs of the treatment of adults and children with allergic rhinitis with or without AIT: already from the eighth month of SCIT onward, total treatment costs were lower than without AIT [36].

\section{Future}

New modalities of AIT are under investigation, although none of them has been tested in children yet, recombinant allergens, hypoallergenic recombinants, Tstimulating peptides, and combination molecules, to name some [41]. Also, new administration routes are sought: some of which seem promising, 
intralymphatic [42] and epicutaneous [43], and in 2014, a protocol for a DBPC trial of bi-monthly intradermal injections of nanogram amounts of grass pollen extract AIT in adults with moderate-severe hay fever was published [44].

\title{
Compliance with Ethics Guidelines
}

\section{Conflict of Interest}

Désirée Larenas Linnemann declares that she has no conflict of interest.

\author{
Human and Animal Rights and Informed Consent
}

This article does not contain any studies with human or animal subjects performed by any of the authors.

\section{References and Recommended Reading}

1. Larenas-Linnemann D, Michels A, Dinger H, ShahHosseini K, Mosges R, Arias-Cruz A, et al. Allergen sensitization linked to climate and age, not to intermittent-persistent rhinitis in a cross-sectional cohort study in the (sub)tropics. Clinical and translational allergy. 2014;4:20.

2. Larenas-Linnemann D, Michels A, Dinger H, AriasCruz A, Ambriz Moreno M, Bedolla Barajas M, et al. In the (sub)tropics Allergic Rhinitis and Its Impact on Asthma classification of allergic rhinitis is more useful than perennial-seasonal classification. American journal of rhinology \& allergy. 2014;28(3):232-8.

3. Portnoy J, Miller JD, Williams PB, Chew GL, Miller JD, Zaitoun F, et al. Environmental assessment and exposure control of dust mites: a practice parameter. Ann Allergy Asthma Immunol. 2013;111(6):465-507.

4. Phipatanakul W, Matsui E, Portnoy J, Williams PB, Barnes C, Kennedy K, et al. Environmental assessment and exposure reduction of rodents: a practice parameter Ann Allergy Asthma Immunol. 2012;109(6):375-87.

5. Larenas-Linnemann D, Mayorga-Butron JL, SanchezGonzalez A, Ramirez-Garcia A, Medina-Avalos M, Figueroa-Morales MA, et al. ARIA Mexico 2014. Adaptation of the Clinical Practice Guide ARIA 2010 for Mexico. Methodology ADAPTE. Revista Alergia Mexico. 2014;61 Suppl 1:S3-116.

6. Peters AT, Spector S, Hsu J, Hamilos DL, Baroody FM, Chandra RK, et al. Diagnosis and management of rhinosinusitis: a practice parameter update. Ann Allergy Asthma Immunol. 2014;113(4):347-85.

7. Cox LS, Casale TB, Nayak AS, Bernstein DI, Creticos PS, Ambroisine L, et al. Clinical efficacy of 300IR 5-grass pollen sublingual tablet in a US study: the importance of allergen-specific serum IgE. J Allergy Clin Immunol. 2012;130(6):1327-34 e1.
8. Portnoy J, Kennedy K, Sublett J, Phipatanakul W, Matsui E, Barnes C, et al. Environmental assessment and exposure control: a practice parameter-furry animals. Ann Allergy Asthma Immunol. 2012;108(4):223 e1-15.

9. Dondi A, Tripodi S, Panetta V, Asero R, Businco AD, Bianchi A, et al. Pollen-induced allergic rhinitis in 1360 Italian children: comorbidities and determinants of severity. Pediatric allergy and immunology : official publication of the European Society of Pediatric Allergy and Immunology. 2013;24(8):742-51.

10. Sedaghat AR, Phipatanakul W, Cunningham MJ. Prevalence of and associations with allergic rhinitis in children with chronic rhinosinusitis. International journal of pediatric otorhinolaryngology. 2014;78(2):343-7.

11. Sedaghat AR, Phipatanakul W, Cunningham MJ. Characterization of aeroallergen sensitivities in children with allergic rhinitis and chronic rhinosinusitis. Allergy \& rhinology. 2014;5(3):143-5.

12. Lariou MS, Dikalioti SK, Dessypris N, Baka M, Polychronopoulou S, Athanasiadou-Piperopoulou F, et al. Allergy and risk of acute lymphoblastic leukemia among children: a nationwide case control study in Greece. Cancer epidemiology. 2013;37(2):146-51.

13. Murphy K, Gawchik S, Bernstein D, Andersen J, Rud PM. A phase 3 trial assessing the efficacy and safety of grass allergy immunotherapy tablet in subjects with grass pollen-induced allergic rhinitis with or without conjunctivitis, with or without asthma. Journal of negative results in biomedicine. 2013;12(1):10.

14. Tosca M, Sivestri M, Accogli A, Rossi GA, Ciprandi G. Serum-specific IgE and allergen immunotherapy in allergic children. Immunotherapy. 2014;6(1):29-33.

15. Larenas-Linnemann D, Pfaar O. Patient-reported outcomes and quality-of-life questionnaires in the assessment of rhinoconjunctivitis in childhood. Current 
opinion in allergy and clinical immunology.

2014;14(3):192-9.

16. Makatsori M, Pfaar O, Calderon MA. Allergen immunotherapy: clinical outcomes assessment. The journal of allergy and clinical immunology In practice. 2014;2(2):123-9.

17. Roder E, Berger MY, Hop WC, de Groot H, van Wijk RG. The relevance of patient-reported outcomes in a grass pollen immunotherapy trial in children and adolescents with rhinoconjunctivitis. Pediatr Allergy Immunol. 2013;24(1):39-48.

18. Rosewich M, Arendt S, El Moussaoui S, Schulze J, Schubert R, Zielen S. Bronchial allergen provocation: a useful method to assess the efficacy of specific immunotherapy in children. Pediatr Allergy Immunol. 2013.

19. Lin SY, Erekosima N, Kim JM, Ramanathan M, SuarezCuervo C, Chelladurai Y, et al. Sublingual immunotherapy for the treatment of allergic rhinoconjunctivitis and asthma: a systematic review. JAMA. 2013;309(12):1278-88.

20. Durham SR, Scadding GW, Phippard D, Wurtzen PA, Shamji MH. Biomarkers of tolerance in response to allergen immunotherapy. Arbeiten aus dem Paul-Ehrlich-Institut. 2013;97:75-81.

21. Shamji MH, Ljorring C, Wurtzen PA. Predictive biomarkers of clinical efficacy of allergen-specific immunotherapy: how to proceed. Immunotherapy. 2013;5(3):203-6.

22. Shamji MH, Francis JN, Wurtzen PA, Lund K, Durham SR, Till SJ. Cell-free detection of allergen-IgE crosslinking with immobilized phase CD23: inhibition by blocking antibody responses after immunotherapy. J Allergy Clin Immunol. 2013;132(4):1003-5 e1-4.

23. Soyer OU, Akdis M, Ring J, Behrendt H, Crameri R, Lauener R, et al. Mechanisms of peripheral tolerance to allergens. Allergy. 2013;68(2):161-70.

24. Narkus A, Lehnigk U, Haefner D, Klinger R, Pfaar O, Worm M. The placebo effect in allergen-specific immunotherapy trials. Clinical and translational allergy. 2013;3(1):42.

25. Larenas-Linnemann DE, Pietropaolo-Cienfuegos DR, Calderon MA. Evidence of effect of subcutaneous immunotherapy in children: complete and updated review from 2006 onward. Ann Allergy Asthma Immunol. 2011;107(5):407-16 e11.

26. Kim JM, Lin SY, Suarez-Cuervo C, Chelladurai Y, Ramanathan M, Segal JB, et al. Allergen-specific immunotherapy for pediatric asthma and rhinoconjunctivitis: a systematic review. Pediatrics. 2013;131(6):1155-67.

27. Rank M, Calabria C, Hauswirth D, Sher L, Larenas LD. AAAAI membership experience with allergen immunotherapy (AIT) safety in young children and pregnant women. J Allergy Clin Immunol. 2013;131(2 suppl):AB186.

28. Vance GH, Goldring S, Warner JO, Cox H, Sihra B, Hughes $\mathrm{S}$, et al. A national audit of pollen immunotherapy for children in the United Kingdom: patient selection and programme safety. Clin Exp Allergy. 2011;41(9):1313-23.

29. Cox L, Larenas-Linnemann D, Lockey RF, Passalacqua G. Speaking the same language: The World Allergy Organization Subcutaneous Immunotherapy Systemic Reaction Grading System. J Allergy Clin Immunol. 2010;125(3):569-74, 74 e1-74 e7.

30. Lucendo AJ, Arias A, Tenias JM. Relation between eosinophilic esophagitis and oral immunotherapy for food allergy: a systematic review with meta-analysis. Ann Allergy Asthma Immunol. 2014;113(6):624-9.

31. Miehlke S, Alpan O, Schroder S, Straumann A. Induction of eosinophilic esophagitis by sublingual pollen immunotherapy. Case reports in gastroenterology. 2013;7(3):363-8.

32. Passalacqua G, Baena-Cagnani CE, Bousquet J, Canonica GW, Casale TB, Cox L, et al. Grading local side effects of sublingual immunotherapy for respiratory allergy: speaking the same language. J Allergy Clin Immunol. 2013.

33. Johnstone DE, Crump L. Value of hyposensitization therapy for perennial bronchial asthma in children. Pediatrics. 1961;27:39-44.

34. Swamy RS, Reshamwala N, Hunter T, Vissamsetti S, Santos CB, Baroody FM, et al. Epigenetic modifications and improved regulatory $\mathrm{T}$-cell function in subjects undergoing dual sublingual immunotherapy. J Allergy Clin Immunol. 2012;130(1):215-24 e7.

35. Amar SM, Harbeck RJ, Sills M, Silveira LJ, O'Brien H, Nelson HS. Response to sublingual immunotherapy with grass pollen extract: monotherapy versus combination in a multiallergen extract. J Allergy Clin Immunol. 2009;124(1):150-6 e1-5.

36. Hankin CS, Cox L, Bronstone A, Wang Z. Allergy immunotherapy: reduced health care costs in adults and children with allergic rhinitis. J Allergy Clin Immunol. 2013;131(4):1084-91.

37. Kiel MA, Roder E, Gerth van Wijk R, Al MJ, Hop WC, Rutten-van Molken MP. Real-life compliance and persistence among users of subcutaneous and sublingual allergen immunotherapy. J Allergy Clin Immunol. 2013;132(2):353-60 e2.

38. Gonzalez-de-Olano D, Alvarez-Twose I. Adherence to immunotherapy in times of financial crisis. Ann Allergy Asthma Immunol. 2013;110(6):466-8.

39. Passalacqua G, Baiardini I, Senna G, Canonica GW. Adherence to pharmacological treatment and specific immunotherapy in allergic rhinitis. Clin Exp Allergy. 2013;43(1):22-8.

40. Larenas Linnemann DE, Blaiss MS. Selection of patients for sublingual versus subcutaneous immunotherapy. Immunotherapy. 2014;6(7):871-84. doi:10. 2217/imt.14.55.

41. Nelson HS. New forms of allergy immunotherapy for rhinitis and asthma. Allergy Asthma Proc. 2014;35(4):271-7.

42. Senti G, Crameri R, Kuster D, Johansen P, MartinezGomez JM, Graf N, et al. Intralymphatic 
immunotherapy for cat allergy induces tolerance after only 3 injections. J Allergy Clin Immunol.

2012;129(5):1290-6.

43. Senti G, Graf N, Haug S, Ruedi N, von Moos S, Sonderegger $\mathrm{T}$, et al. Epicutaneous allergen administration as a novel method of allergen-specific immunotherapy. J Allergy Clin Immunol. 2009;124(5):997-1002.
44. Slovick A, Douiri A, Kelly J, Guerra A, Muir R, Tsioulos $\mathrm{K}$, et al. Protocol for a double-blind randomised controlled trial of low dose intradermal grass pollen immunotherapy versus a histamine control on symptoms and medication use in adults with seasonal allergic rhinitis (PollenLITE). Clinical and translational allergy. 2013;3(1):27. 